This document is the unedited Author's version of a Submitted Work that was subsequently accepted for publication in ACS Applied Materials and Interfaces, copyright (c) American Chemical Society after peer review. To access the final edited and published work see:

https://dx.doi.org/10.1021/acsami. 8b19062. 


\section{Role of Penetrability into a Brush-coated Surface in Directed Self-Assembly of Block Copolymers}

Laura Evangelio ${ }^{1,2}$, Marta Fernández-Regúlez', Jordi Fraxedas ${ }^{2}$, Marcus Müller ${ }^{3}$, Francesc PérezMurano $^{1, *}$

${ }^{1}$ Institute of Microelectronics of Barcelona (IMB-CNM, CSIC). Campus de la UAB, 08193, Bellaterra, Spain

${ }^{2}$ Catalan Institute of Nanoscience and Nanotechnology (ICN2), CSIC and BIST, Campus UAB, Bellaterra, 08193 Barcelona, Spain

${ }^{3}$ Institute for Theoretical Physics, Georg-August University, Friedrich-Hund-Platz 1, 37977 Göttingen, Germany

*francesc.perez@imb-cnm.csic.es

\section{KEYWORDS}

Block copolymer, directed self-assembly, chemical epitaxy, interface energy, wide stripes 


\begin{abstract}
High density and high resolution line and space patterns on surfaces are obtained by directed selfassembly of lamella-forming block copolymers using wide-stripes chemical guiding patterns. When the width of the chemical pattern is larger than the half-pitch of the block copolymer, the interaction energy between each block copolymer domain and the surface is crucial to obtain the desired segregated film morphology. We investigate how the intermixing between block copolymers and polymer brush molecules at the surface influences the optimal surface and interface free energies to obtain a proper block copolymer alignment. We have found that computational models successfully predict the experimentally obtained guided patterns if the penetrability of the brush layer is taken into account instead of a hard, impenetrable surface. Experiments on directed self-assembly of lamella-forming PS-b-PMMA using chemical guiding patterns corroborate the models used in the simulations, where the values of the surface free energy between block copolymer and guiding and background stripes are accurately determined using an experimental method based on the characterization of contact angles in droplets formed after dewetting of homopolymer blends.
\end{abstract}




\section{INTRODUCTION}

For many decades, miniaturization as defined by Moore's Law has dictated the evolution of the microelectronic industry bound to provide integrated circuits of enhanced functionality and performance while decreasing production costs. ${ }^{1}$ At present, the critical dimensions required for the new generation of integrated circuits forcedly implies new challenges; hence new fabrication strategies need to be considered.

Directed self-assembly (DSA) of block copolymers (BCPs) is becoming a very promising alternative lithographic technique for the creation of nanometer-scale patterns due to its high throughput and process simplification compared to other approaches. ${ }^{2-5}$ Several recent studies have shown that DSA can improve the line-width roughness $(\mathrm{LWR})^{6}$ and line-edge roughness (LER) ${ }^{7}$ at very high patterning resolutions. Block copolymers are macromolecules composed of two or more than two kinds of chemically distinct homopolymers linked end-to-end by covalent bonds. When the different blocks are chemically immiscible, the balance between entropically and enthalpically driven phase separation and the chemical bond constraints between the blocks drives the formation of ordered micro-domains. The phase behavior is determined by three factors: the overall degree of polymerization, $N$, the volume fraction of one of the blocks in a diblock copolymer, $f$, and the Flory-Huggins interaction parameter, $\chi$, which quantifies the repulsive interaction between the blocks. The strength of the segregation power is dictated by the reduced parameter $\chi N$, which should exceed a critical value in order to have micro-phase segregation, i.e., $\chi N=10.5$ according to mean field theory for a symmetric $(f=0.5)$ block copolymer. Block copolymers of low molecular weight have a low value of $N$, which induces the decline of the value of $\chi N$ below the minimum value to micro-phase segregate. ${ }^{8}$

Lamella-forming BCPs are one of the most interesting morphologies from a technological point of view. After self-assembly, lamellae can be oriented perpendicular or parallel to the substrate, depending on whether the interaction strength between the substrate and the BCP is similar for both blocks or stronger 
to one of them, respectively. Therefore, a proper wetting balance of the polymer blocks to the substrate is needed to promote lamellae oriented perpendicular to the substrate. The most common way to control the orientation of the BCP micro-domains consists in using neutral surfaces to control the surface free energy, so that the difference in the surface interaction of the two blocks is small.. ${ }^{9}$

Thin films of vertical lamellae form patterns whose morphology is randomly oriented; the width of the lines being defined by the molecular weight of the BCP. Nevertheless, ordered arrays of lines with a predefined orientation and registered with respect to device boundaries are required for most of technological applications, not only for high volume manufacturing, but also as a general method to realize templates for anisotropic metal nanostructures ${ }^{10}$. This can be achieved by pre-structuring the substrate with guiding patterns of appropriate dimensions and shape. These guiding patterns are realized by grapho- or chemoepitaxy methods. In graphoepitaxy, ${ }^{11-13}$ the guiding patterns are made of topographical features. Usually, the width of the trench becomes a limitation for achieving high density of integration. ${ }^{14-16}$

Therefore, in order to avoid this limitation, chemical epitaxy methods have been intensively explored to produce highly dense patterns. ${ }^{3,17-19}$ Moreover, in chemical epitaxy there is a reduction of the edge roughness due to the self-healing of the $\mathrm{BCP}$, which means that the irregularities of the guiding patterns are not transferred to the $\mathrm{BCP}$ pattern. ${ }^{20}$ Chemical guiding patterns are fabricated by conventional topdown lithographic methods like optical, extreme ultraviolet (EUV) projection or electron beam lithography (EBL), in combination with a selective, local chemical modification of the brush surface layer.

Usually, chemical guiding patterns are formed by the combination of guiding stripes that have strong affinity for one domain, and background stripes which present neutral or almost neutral affinity for both BCP domains. When the surface has strong affinity for one of the domains, the width of guiding stripes should be approximately half of the pitch of the BCP, if one wants to avoid a parallel, lying lamellar morphology. In consequence, high accuracy and high resolution lithography methods are needed for the definition of guiding patterns, increasing thus the process complexity and cost. ${ }^{3,21,22}$ 
An alternative patterning strategy is based on using wider guiding stripes, which present slightly affinity for one block. In this way, large density multiplication factors (defined as the relation between the pitch of the guiding pattern and the pitch of the DSA pattern) can be obtained. Here, we investigate under which conditions guiding stripes with width equal to $(n+0.5) L_{0}$ (with $n \geq 1$ and $L_{0}$ being the lamellar periodicity) can efficiently guide the self-assembly when the interface energies are tuned adequately. There are several works which employ this guiding pattern strategy. ${ }^{22-29}$

Previous investigations have shown that the optimal guiding pattern conditions depends on both the dimensions of the guiding patterns and the chemistry of interactions with the BCP domains occurring at the surface of the chemical guiding patterns. ${ }^{19}$ In consequence, the achievement of the suitable block copolymer alignment with high-density multiplication factors by chemical epitaxy using wide guiding stripes requires considering multiple parameters. The prediction of their optimal value is complex due to the involvement of many inter-related phenomena. The most significant parameters are of dimensional nature (BCP film thickness, $D$, and guiding pattern dimensions), materials related (number of statistical segments per diblock copolymer, $N$, the statistical segment length, $b$, and the Flory-Huggins parameter, $\chi)$ and process related (annealing temperature, solvent material, solution concentration and interface boundary energies). In addition to the here-referred parameters, the chemical interactions that take place between each domain of the BCP and the patterned surface (guiding and background stripes) is of prime importance as the main driving force in DSA by chemical epitaxy ${ }^{30}$. These interactions, in turn, are related to the difference of surface free energies of each copolymer domain and the confining boundary, $\Delta \gamma=\gamma_{\mathrm{SA}}$ $-\gamma_{\mathrm{SB}}$

On the other hand, computational, top-down models of DSA have proven useful to explore the large parameter space spanned by thermodynamic conditions, like incompatibility and surface affinity, as well as process conditions. The use of these coarse-grained models relies on the identification of a small number of coarse-grained parameters, i.e., the chains' end-to-end distance, $R_{\ell}$, the composition of the block copolymer, and the incompatibility, $\chi N$, between blocks. The physical behavior depends on the 
detailed material properties only via these coarse-grained parameters, which, in turn, can be obtained from independent experimental measurements. These top-down models can successfully and quantitatively predict the kinetics of DSA. ${ }^{31-34}$ In the spirit of a top-down strategy, the surface affinities of the guiding stripes and neutral background are often modeled by an impenetrable surface with short-range attraction. The strength of the interaction is chosen as to reproduce the coarse-grained parameter, $\Delta \gamma$, but the detailed molecular mechanism that gives rise to a surface free-energy difference is often assumed not to be relevant. Density multiplication with wide guiding stripes is a demanding test of modeling because successful DSA only occurs in a narrow parameter window of surface affinities. On the one hand, the wide guiding stripe must present sufficiently strong affinity for one block in order to direct the structure formation. On the other hand, a too strong affinity will result in lying lamellae on top of the guiding stripes. $^{29}$

Knowing the properties of the brush layer is key to investigate the characteristic of the self-assembly process. Previous works include the influence of the grafting temperature of the brush layer ${ }^{35}$, its thickness $^{36}$ and the mechanical properties ${ }^{37}$. Grafting density has been found to improve the thickness process window for perpendicular orientation of lamellar on free surfaces.

Here, we introduce the effect of brush penetrability on the alignment of PS-b-PMMA lamellae forming block copolymers by chemical guiding patterns with wide guiding stripes. The presence of block copolymer / brush intermixing has being pointed out previously ${ }^{9}$. We include this effect in computer simulations to obtain the conditions that sustain standing lamella in chemical epitaxy. The experimental values of the free surface energies of the chemical guiding patterns are included in the model and they need to be determined with high accuracy. Because of this, we have employed an experimental method based on the characterization of the contact angle in droplets formed by de-wetting of thin layers of homopolymer blends forming a droplet-in-droplet structure. 


\section{RESULTS AND DISCUSSION}

\section{a) Chemical epitaxy DSA using wide guiding stripes}

Figure 1.a illustrates the process to direct the self-assembly of poly(styrene-block-methyl methacrylate) (PS- $b$-PMMA) of $22 \mathrm{~nm}$ pitch using wide guiding stripes. Chemical guiding patterns are created on silicon substrates by first depositing a $8.6 \mathrm{~nm}$ thick layer of $70 \%$ rich poly(styrene-random-methyl methacrylate) (PS $70 \%-r$-PMMA) brush. Then a layer of $80 \mathrm{~nm}$ of PMMA is patterned by EBL, and after the development, the sample is exposed to oxygen plasma to chemically modify the defined regions. The conditions under which the brush is exposed to oxygen plasma are critical to obtain efficient guiding patterns. We have investigated two different conditions, which are described in the materials and methods sections; they are referred to as mild ( $150 \mathrm{~W}, 10 \mathrm{sccm} \mathrm{O}_{2}$ for 5 seconds) and strong ( $300 \mathrm{~W}, 50 \mathrm{sccm} \mathrm{O}_{2}$ for 10 seconds) oxygen plasma conditions. The resulting chemical guiding patterns, after removing the electron beam resist, have a guiding stripe width of $1.5 L_{0}$ and a period equal to $n L_{0}$. AFM characterization before BCP deposition reveals a chemical contrast without almost any change in topography for the mild oxygen plasma conditions (the oxygen plasma exposure removes less than $0.5 \mathrm{~nm}$ of the brush layer). (See supplemental information, figure S1). Surface characterization of the brush layer reveals an increased affinity of the brush layer with respect to PMMA when it is exposed to oxygen plasma ${ }^{30}$ and, under mild oxygen plasma conditions, a very low damage (See the AFM characterization of the chemically functionalized guiding patterns in the supplemental information, figure S1).

Then, PS- $b$-PMMA is deposited and annealed at $230^{\circ} \mathrm{C}$ for $10 \mathrm{~min}$ with a continuous nitrogen flow. The typical thickness, $D$, of the PS- $b$-PMMA layer is $3 L_{0} / 4$. The dependence of block copolymer orientation on the layer thickness is complex and related to the surface wetting behavior ${ }^{38}$. This thickness maximizes the geometric frustration of lying lamellae, ${ }^{39,40}$ and thereby allows to make compatible a strong enough surface affinity for one block while keeping a standing lamellae morphology. 
Figures 1.b-d show SEM images of aligned PS- $b$-PMMA of $22 \mathrm{~nm}$ pitch with density multiplication factors of 3, 4 and 5, respectively. In all cases, the guiding stripe width is fixed to $1.5 L_{0}$. For guiding patterns with pitch larger than $5 L_{0}$, the $\mathrm{BCP}$ alignment is lost on the background stripes. The same method for guiding pattern generation has also been applied to align PS- $b$-PMMA BCP of $28 \mathrm{~nm}$ and $38 \mathrm{~nm}$ pitch. A good alignment using wide stripes is also achieved, adapting the processing conditions and brush layers to each BCP material (see supplemental information, figure S2). In the following, we center our analysis to the case of alignment of PS-b-PMMA of $11 \mathrm{~nm}$ half-pitch, as this is the material with higher technological interest because it delivers higher resolution features.

\section{b) Experimental determination of surface free energies}

The understanding of the guiding mechanism requires a precise knowledge of multiple parameters, among which the difference of surface free energies between the substrate and each BCP domain, $\Delta \gamma$, plays a dominant role. Next, we show that an accurate value of the surface free energy difference can be obtained by the contact angle, $\theta_{\mathrm{A}-\mathrm{B}}$ that the $\mathrm{AB}$ interface of an homopolymer blend makes with the brush-coated surface. We use this value to quantitatively match the simulation model to the experimental system. Additionally, and independently, the contact angle can be employed in conjunction with a simple geometrical model to start analyzing the conditions to obtain free standing lamellae.

The experimental method to determine the value of the interfacial energies is based on performing homopolymer blend de-wetting experiments, ${ }^{41,42}$ and measuring the contact angle, $\theta_{\mathrm{A}-\mathrm{B}}$, of the internal interface between both homopolymers at the brush surface. ${ }^{43}$ This method is an extension of the method proposed by Mansky et al. ${ }^{9}$ It allows to directly determining $\Delta \gamma$ from the experimental measure of one single angle, and, as it is shown below, it is more accurate.

The de-wetting experiment is performed as follows: a 50:50 PS/PMMA homopolymer blend is spincoated on the top of the brush, and it is subsequently annealed under the same conditions as for the DSA process. After the annealing, if the substrate is not attractive enough to at least one of the homopolymers, 
a de-wetting phenomenon occurs, forming a drop-in-drop structure, which contains both homopolymers (figure 2.a). The angle $\theta_{\mathrm{A}-\mathrm{B}, \text { that }}$ the internal interface between both homopolymers forms at the contact line with the brush provides direct evidence of the difference of surface free energy, $\Delta \gamma$. If $\theta_{\mathrm{A}-\mathrm{B}}<90^{\circ}$, then the surface has stronger affinity for homopolymer $\mathrm{B}$, and if $\theta_{\mathrm{A}-\mathrm{B}}=90^{\circ}$, then the surface presents equal affinity for both homopolymers. SEM images of PS/PMMA polymer blend droplets on top of un-modified and modified (mild) PS $_{70 \%}$-r-PMMA brush layers after removing PMMA domains are shown in figure 2.b. and 2.c, respectively. The change of the affinity character of the surface is made evident by the change of the morphology of the droplets and by the change of contact angle. To get a representative value of the contact angle we have measured and averaged the values from five different droplet structures for each material combination and processing conditions.

By applying Young's equation to the specific case of PS/PMMA blends, we directly relate the surface affinity to the observed contact angle and the interface tension between the blocks:

$\Delta \gamma=\gamma_{\text {Brush-PS }}-\gamma_{\text {Brush-PMMA }}=\gamma_{\text {PS/PMMA }} \cdot \cos \theta_{\mathrm{PS} / \mathrm{PMMA}}$,

where $\gamma_{\text {Brush-PS }}$ and $\gamma_{\text {Brush-PMMA }}$ are the surface tensions between each homopolymer and the brush-coated surface, and $\gamma_{\mathrm{PS} / \mathrm{PMMA}}$ is the interface tension between PS and PMMA, which slightly depends on the annealing temperature and can be obtained from literature: ${ }^{44}$

$\gamma_{\mathrm{PS} / \mathrm{PMMA}}[\mathrm{mN} / \mathrm{m}]=3.6-0.013 \mathrm{~T}\left[{ }^{\circ} \mathrm{C}\right]$,

Equations [1] and [2] show that the difference of surface tension of PS and PMMA with the brush is directly obtained from the measurement of the contact angle of the PS/PMMA interface.

Previous works have used de-wetting experiments to determine surface affinity, but not using homopolymer blends as we propose here. ${ }^{45-48}$ The advantage of the homopolymer blend method is that it provides an information (contact angle between the homopolymers at the brush surface) which can be directly fed into the simulations. Compared to the method proposed by Mansky et $\mathrm{al}^{9}$ in which, the surface 
energies are obtained from independent dewetting experiments of homopolymers, the present method is about an order of magnitude more accurate because the relevant difference of surface energies is not calculated as the difference between two large numbers but directly, as the PS-PMMA interface tension is about one order of magnitude smaller than the surface tensions of both polymers (as it is presented below). Additionally, we have to point out that the present method works even if one of the both components wet the brush, while Mansky's method requires that the homopolymer films dewet.

According to Mansky et al., the contact angle $\theta_{i}$ between one homopolymer $\mathrm{i}$ and the brush is determined by Young's equation:

$$
\gamma_{b}=\gamma_{h_{i}} \cos \left(\theta_{i}\right)+\gamma_{b h_{i}}[3]
$$

where:

$\gamma_{b}$ is the surface tension of the brush;

$\gamma_{h_{i}}$ is the surface tension of homopolymer $i$;

$\gamma_{b h_{i}}$ is the surface tension between brush and homopolymer $i$;

The difference of surface tension $(\Delta \gamma)$ between the brush and two different pure homopolymers $\left(\mathrm{h}_{1}\right.$ and $\mathrm{h}_{2}$ ) can be obtained by performing two different de-wetting experiments, and then calculated as follows:

$\Delta \gamma=\gamma_{b h_{1}}-\gamma_{b h_{2}}=-\gamma_{h_{1}} \cos \left(\theta_{1}\right)+\gamma_{h_{2}} \cos \left(\theta_{2}\right) \approx \gamma_{h_{i}}\left(\cos \left(\theta_{2}\right)-\cos \left(\theta_{1}\right)\right)[4]$

Typical values $\gamma_{h_{i}}$ of PS and PMMA are very similar and the resulting difference of surface tension is usually below $1 \mathrm{mN} / \mathrm{m}^{9}$. This makes that any inaccuracy on the determination of $\left(\cos \left(\theta_{2}\right)-\cos \left(\theta_{1}\right)\right)$ is amplified in the calculation of $\Delta \gamma$.

On the contrary, when $\Delta \gamma$ is calculated from the contact angle between the two homopolymers in blend dewetting experiments, the uncertainty is lower, as it is calculated from:

$$
\Delta \gamma=\gamma_{12} \cos \left(\theta_{12}\right)[5]
$$

where $\gamma_{12}$ is the interface tension between the coexisting phases of the binary homopolymer system, and, so, it is of the same order of magnitude of $\Delta \gamma$.

The uncertainty in the calculation of $\Delta \gamma, \varepsilon_{i}(\Delta \gamma)$, can be calculated, assuming that the uncertainty is only present in the determination of the angles, as follows: 
i) $\quad \varepsilon_{i}(\Delta \gamma)=\gamma_{h_{i}} \sqrt{\sin ^{2}\left(\theta_{1}\right)+\sin ^{2}\left(\theta_{2}\right)} \varepsilon\left(\theta_{i}\right)$ for the case of droplets of homopolymers

ii) $\quad \varepsilon_{i i}(\Delta \gamma)=\gamma_{h_{12}} \sin \left(\theta_{12}\right) \varepsilon\left(\theta_{12}\right)$ for the case of the droplet of a blend of homopolymers

which clearly shows that the uncertainty using the methods of homopolymers blends is more accurate:

$$
\frac{\varepsilon_{i}(\Delta \gamma)}{\varepsilon_{i i}(\Delta \gamma)}=\frac{\gamma_{h_{i}} \sqrt{\sin ^{2}\left(\theta_{1}\right)+\sin ^{2}\left(\theta_{2}\right)}}{\gamma_{h_{12}} \sin \left(\theta_{12}\right)} \approx 0.6 \frac{\gamma_{h_{i}}}{\gamma_{h_{12}}}[6]
$$

Taking a specific quantitative situation for one of the systems investigated here (brush PS $_{70 \%}$-r-PMMA exposed to an oxygen plasma using mild conditions):

$\gamma_{h_{i}}=25.3 \mathrm{mN} / \mathrm{m}$ (for both, PS and PMMA); $\gamma_{12}=0.61 \mathrm{mN} / \mathrm{m} ; \theta_{1}=12.5 ; \theta_{2}=5^{\circ} ; \theta_{12}=146.4^{\circ}$ yields to the following results (assuming un uncertainty of $5^{\circ}$ in the determination of the angles):

i) $\Delta \gamma=-0.5 \mathrm{mN} / \mathrm{m} \pm 0.52$ for the case of droplets of homopolymers

ii) $\Delta \gamma=-0.5 \mathrm{mN} / \mathrm{m} \pm 0.03$ for the case of the droplet of a blend of homopolymers

Tables 1 summarize the results of contact angles for the different material systems and processing conditions that have been investigated here (the values depict the average and standard deviation of the five images analyzed for each situation). Table 1 shows the values of the contact angle $\theta_{\mathrm{A}-\mathrm{B}}$ for the processing conditions used to align PS-b-PMMA of $22 \mathrm{~nm}$ pitch (i.e., (T=230 ${ }^{\circ} \mathrm{C}$, which implies that the value of $\gamma_{\mathrm{PS} / \mathrm{PMMA}}$ is $0.61 \mathrm{mN} / \mathrm{m}$ as stated in equation 2). It shows that a PS percentage higher than $60 \%$ in the PS- $r$-PMMA brush is required to have an almost neutral surface $(\Delta \gamma \approx 0)$, as corroborated experimentally by the fact that this condition has to be fulfilled to obtain perpendicular lamellae when no chemical guiding patterns are present (see supplemental information, figure S3). Table S2 in the supplemental information provides complementary data of the contact angle determination for other combinations of brush layer and annealing conditions. Figure S4 in the supplemental information provides the value of water contact angle for the investigated brush layers.

Another method to obtain information from the interfacial energies is by measuring the contact angle with the sessile drop method ${ }^{49}$. It is interesting to compare the results obtained from this method with the ones obtained from the de-wetting experiments in order to check their consistency. In order to determine the 
surface free energy from the contact angle, at least two liquids with known disperse and polar components of surface tensions are required. Nevertheless, the sessile drop method provides the surface free energy of the brush, but not directly the difference of surface free energies between the BCP domains. Therefore, we use the value of the difference of interface energies, obtained from homopolymer blends de-wetting experiments, in the simulations. Figure S4 and table S2, in the supplemental information, show that the water-contact-angle dependence on surface functionalization follows the same trend than the contact angles determined from the de-wetting experiments of homopolymer blends.

\section{c) Geometrical model to relate contact angle with surface free energies}

In the case of thin films of block copolymers, neglecting any effect from the arrangement of the copolymers as they uniform fill space, we assume that the internal AB interfaces of perpendicular lamellae are characterized by the homopolymer contact angle, $\theta_{\mathrm{A}-\mathrm{B}}$, with the substrate. The contact angle arises from a force balance, i.e., Young's law, and in the ultimate vicinity of the three-phase contact line (Adomain, B-domain, brush) the forces in homopolymer blend and diblock copolymer are similar. Moreover, previous work on thin films indicated that, in the case of pinned three-phase contacts, the internal $\mathrm{AB}$ interfaces of perpendicular lamellae are only slightly curved and the domains adopt a shape between ideal wedges and domains with vertical, internal AB interfaces ${ }^{50}$ For simplicity, we assume an ideal-wedge geometry in the following, and this simple geometric construction results in the condition

$$
\tan \theta_{\mathrm{A}-\mathrm{B}}>2 D / L_{0}
$$

for vertical lamellae, where $L_{0}$ is the lamellar period and $D$ the thickness of the copolymer layer. Given that the ideal-wedge geometry is only an approximation, this relation tends to slightly underestimate the maximally permissible surface preference. Using the optimal value, $D=3 L_{0} / 4$, which maximally frustrates lying lamellae, we obtain the condition $\tan \theta_{\mathrm{A}-\mathrm{B}}>3 / 2$ or $123.7^{\circ}>\theta_{\mathrm{A}-\mathrm{B}}>56.3^{\circ}$. 
Specifically, for PS-b-PMMA of $22 \mathrm{~nm}$ pitch and PS $70 \%-r$-PMMA, it is observed that the background regions have stronger affinity for PS domains. After the oxygen plasma functionalization (i.e. guiding stripes) the surface presents higher affinity for PMMA. However, the contact angle observed is (see Table 1) above the value that guarantees standing lamellae according to the geometrical model mentioned above (see Eq. (7)).

\section{d) Simulations}

To understand the origin of this discrepancy, we investigate by means of computer simulations the formation of standing lamellae in chemical epitaxy when using wide guiding stripes. First, we consider the substrate as an impenetrable surface (hard-wall model) with short-ranged interactions, whose strength is tailored to yield the same contact angle as the de-wetting experiment. In a coarse-grained model, the strength of the affinity for wetting is characterized by the parameter $\Lambda N$. In the strong-segregation regime, $\chi N>>10,{ }^{51}$ and assuming a uniform density profile at the wall and an identical entropy loss of the A and B chains as they pack against the surface, we can approximately relate the affinity parameter $\Lambda N$ to the contact angle, $\theta_{\mathrm{A}-\mathrm{B}}$

$\cos \left(\theta_{A B}\right)=\frac{\Delta \gamma}{\gamma_{A B}} \approx \sqrt{\frac{12 \pi}{\chi N}}\left(1-\frac{4 \ln 2}{\chi N}\right)^{-1} \Lambda N=c \Lambda N[8]$

In order to validate the linear relation between the cosine of the contact angle and the surface preference, $\cos \theta_{A B}=c \Lambda N$, and accurately determine the constant of proportionality, $c$, simulations have been performed for a symmetric homopolymer blend (A and B homopolymers). (See methods). The antisymmetric set- up gives rise to a (nearly) straight $\mathrm{AB}$ interface that runs from the bottom to the top surface (see figure 3.a). The minimization of interface curvature allows us to accurately measure the contact angle between the $\mathrm{AB}$ interface and the surface - in contrast to a microscopic droplet set-up, the line tension does not influence the contact angle and the $\mathrm{AB}$ interface is not curved. Figure 3.b presents $\cos \theta_{A B}$ as a function of the strength of the surface interaction $\Lambda N$ as obtained from the simulation data 
(and compares the data with the analytical prediction, (dashed line)). Simple linear fits (solid lines) yield $\cos \theta_{A B}=\mathrm{c} \Lambda N$ with $\mathrm{c}=1.58 ; 1.4$ and 1.15 for $\chi N=16 ; 20$ and 30, respectively. Deviations from the analytical prediction arise from discretization effects of the chain contour and space, and from the fact that the analytical formula is obtained in the strong segregation limit $\chi N>>1$. Figure 3.c shows the result of the simulations by introducing the experimental conditions (materials and dimensions) corresponding to the system shown in figure $1 . d$ (guiding stripe $=1.5 L_{0}$; background stripe $3.5 L_{0}$ ). Simulations predict the formation of parallel, lying lamellae on top of the guiding stripes and vertical lamella on top of the background stripe. This finding is in agreement with the simple geometric estimate, Eq. (7), i.e., the surface preference of the guiding stripes is too strong. The vertical lamellae exhibit a quasi-orthogonal orientation with respect to the guiding pattern orientation because this orientation minimizes the grainboundary free energy of these orthogonal lamellar structures.

To rationalize the discrepancy between the experimental results and the simulations, three effects may be considered: (1) a decrease of the actual value of the difference of surface free energies, (2) the role of surface topography, and (3) considering that the brush layer provides a soft surface, whose properties cannot be entirely quantified by the coarse-grained parameter, $\Delta \gamma$, alone.

(1) A reduction of the surface affinity compared to the de-wetting experiments (without altering the incompatibility inside the film) could possibly result from a temporary enrichment of solvent between substrate and copolymer material in the early stages of the DSA process. There is, however, no direct quantitative experimental evidence for this hypothesis. (2) We have also discarded the effect of surface topography, because the AFM measurements for the guiding patterns do not show appreciable topography for the mild oxygen plasma condition. (3) Recent studies have indicated that (i) a random copolymer brush responds to a contacting liquid by exposing favorable moieties to the interface and thereby lowering the surface tension ${ }^{52,53}$ and (ii) the interpenetration of copolymers into a soft surface tend to stabilize 
perpendicular lamellae. ${ }^{54}$ Therefore, we have explicitly modeled the surface by a random copolymer brush tailoring the brush composition as to match the experimental values of the contact angle.

To provide a more detailed representation of the soft experimental brush surface, we explicitly consider a random copolymer brush in the simulations. In accordance with the experiment, the length of the brush chains is a quarter of length of the copolymers. The random copolymer brush is characterized by two parameters: its composition, $f_{b}$, and its grafting density, $\sigma$. The composition, $f_{b}$, mostly controls the difference, $\Delta \gamma$, of surface tensions and the grafting density, $\sigma$, the penetrability. An extremely high grafting density corresponds to an impenetrable hard surface, whereas intermediate and small grafting densities allow for inter-digitation. Extremely low grafting densities even allow the polymer melt to interact with the non-preferential, impenetrable grafting surface. The effective brush height, $h$, is related to the grafting density, $\sigma$, of the random copolymers via

$$
\frac{h}{R_{e 0}}=\frac{\sigma R_{e 0}^{2} N_{\mathrm{rc}}}{\sqrt{\overline{\mathcal{N}} N}}[9]
$$

Specifically, one end of the random $\mathrm{AB}$ copolymers that form the brush is immobilized at a random lateral position $(y, z)$ on top of a hard, non-preferential grafting surface. The sequence of segments is uncorrelated, and the average fraction of A content of the brush is denoted as $f_{b}$. For simplicity, the A segments of the random copolymer interact like A segments of the copolymer or homopolymer and have identical statistical segment length; likewise, the B segments of the random copolymer are structurally identical to the corresponding copolymer or homopolymer segments.

A typical two-dimensional composition profile of a homopolymer blend between two antisymmetric random-copolymer brushes is presented in figure 4.a. The A and B fractions of the random copolymer brush is switched between the top and bottom, $f_{\mathrm{b}, \text { top }}=1-f_{\mathrm{b}}$,bottom. The antisymmetric setup allows for an accurate determination of the contact angle in a film with rather modest thickness, $D$. 
The dependence of the contact angle $\theta_{A B}$ on the average composition of the random copolymer brush, $f_{b}$, is presented in Figure 4.b for various grafting densities. It is found that (i) $\cos \theta_{A B}$ depends roughly linearly on the composition, and (ii) at a fixed composition, the contact angle decreases with grafting density. Moreover, we note that the homopolymer blend makes a finite contact angle even on a homopolymer brush, $f_{b}=0$ or 1 , because the long homopolymer chains cannot penetrate into the brush, giving rise to a finite surface tension of the brush-homopolymer interface (autophobicity). ${ }^{55,56}$ The contact angle on a pure homopolymer brush, $f_{b}=0$ or 1 , increases with grafting density. For the grafting densities that we have considered, however, $\left|\cos \theta_{\mathrm{AB}}\right|$ remains smaller than 0.9 . Therefore, using the same segment types, $\mathrm{A}$ and $\mathrm{B}$, for the random copolymer brush as for the copolymer we cannot represent the strong plasma condition of the experiment.

The cosines of the experimental contact angles for the soft plasma condition from table I are indicated by the horizontal lines in figure 4.b. Note that due to the structural symmetry between A and B segments, the contact angle, $\theta_{A B}\left(f_{b}\right)$, as a function of brush composition, $f_{b}$, obeys the symmetry relation, $\cos \theta_{A B}\left(1-f_{b}\right)=$ $-\cos \theta_{A B}\left(f_{b}\right)$

For the DSA simulation, presented in figure 4.c, we have chosen an intermediate value of the grafting density ( $a k a$ brush height, cf. Eq. 9) that is compatible with the experimental data and have tailored the composition, $f_{b}$, as to match the experimental contact angle values in table I. As it is shown in figure 4.c, modelling the brush as a soft, penetrable layer of finite thickness and using a composition that results in the experimental contact angles allows that the simulations to reproduce the experimental successful DSA conditions on wide guiding stripes without any adjustment of parameters.

\section{e) Effect of oxygen plasma exposure of the brush layer and critical discussion.}

The affinity of the guiding pattern for the PMMA block can be increased by increasing the exposure to the oxygen plasma. Figure 5 shows a SEM image of a pattern created under strong plasma conditions (guiding stripe width: $1.5 L_{0}$; background stripe width: $3.5 L_{0}$ ). In this case, on top of the guiding stripe the 
block copolymer presents parallel oriented configuration, and on top of the background stripes, the polymer is oriented orthogonal to the direction of the guiding pattern. Similar patterns have been shown before. ${ }^{19,22,57-59}$ This morphology resembles the simulation result in Figure 3.a, indicating that it corresponds to a too strong preference of the guiding pattern. In our model of a random copolymer brush, the segments of the brush and the copolymer are structurally identical and Figure 3.b already employs the maximal preference of the guiding stripes within the random copolymer brush model. In Figure S5 we verify that using an even stronger preference of the guiding stripes for an impenetrable surface with shortrange interaction, which matches the experimental contact angle, does not alter the morphology on top of the guiding stripes.

\section{EXPERIMENTAL}

\section{Materials}

Lamellae-forming poly(styrene-block-methyl methacrylate) (PS- $b$-PMMA) $\left(\mathrm{Mn}=42.3 \mathrm{~kg} \cdot \mathrm{mol}^{-1}\right.$ and PDI $=1.10$ ) has been used as a BCP material, by using a poly(styrene-random-methyl methacrylate) (PS- $r$ PMMA) brush with PS:PMMA volume ratio of 70:30 $\left(\mathrm{Mn}=9.9 \mathrm{~kg} \cdot \mathrm{mol}^{-1}\right.$ and PDI $\left.=1.34\right)$. These materials have been supplied by ARKEMA under the tradename Nanostrenght ${ }^{\circledR}$ in powder, and then dissolved in propylene glycol methyl ether acetate (PGMEA).

For e-beam definition of the chemical guiding patterns, PMMA (polymethyl methacylate) $(950 \mathrm{~kg} / \mathrm{mol}$, dissolved at $2 \%$ in anisole) has been used as a resists. The developer solution are prepared by mixing MIBK (methyl isobutyl ketone): IPA (Isopropanol) ratio 1:3. These products, the e-beam resist and developer, are purchased form MicroChem.

\section{Samples preparation}

The starting substrates are $0.9 \times 0.9 \mathrm{~cm}^{2}$ chips bearing a native silicon oxide layer $((100)$, p-type silicon wafers of 4-40 $\Omega \cdot \mathrm{cm}$ resistivity). Previously to the grafting process, the samples are cleaned and activated 
by oxygen plasma for $10 \mathrm{~min}$ at $500 \mathrm{~W}$ and $50 \mathrm{sccm}$ oxygen flow. This step allows to create OH- groups on the surface that will facilitate the grafting of the brush layer . Oxygen plasma exposure of silicon substrate efficiently remove surface contamination from species containing carbon and oxygen components and reduce the concentration of $\mathrm{Si}-\mathrm{O}$ bonds typically presents on non-stoichiometric $\mathrm{SiOx}$ natural silicon ${ }^{60}$. On the other hand, the plasma also increases the amount of $-\mathrm{OH}$ groups ${ }^{61}$ improved on the surface reaction for polymer grafting of random co-polymers.

In DSA of block copolymers the surface preparation by using an oxygen plasma pre-cleaning before copolymer grafting is a standard procedure ${ }^{4,62}$

Then a 2\% PGMEA brush solution is spin-coated at $5000 \mathrm{rpm}$ on the top of the surface, and annealed at $230^{\circ} \mathrm{C}$ for 5 min in a nitrogen environment. Consequently, the non-grafted brush is removed by dipping the sample in PGMEA for $5 \mathrm{~min}$ in an ultrasonic bath at $40^{\circ} \mathrm{C}$. The brush film thickness grafted after PGMEA rinsing is $8.6 \mathrm{~nm}$ as determined by ellipsometer. Then a $2 \%$ 950k PMMA anisole solution is spin-coated on the top of the brush at $2000 \mathrm{rpm}$ giving rise to an $80 \mathrm{~nm}$ film thickness.

Once the resist has been deposited, the guiding patterns are defined by electron beam lithography (EBL) in a RAITH150Two equipment. Guiding patterns for line/space applications with guiding stripes widths of $1.5 \mathrm{~L}_{0}$ and density multiplication factors of 3 to 5 have been designed. The desired guiding patterns have been defined by using an acceleration voltage of $20 \mathrm{kV}$ and $110 \mathrm{pA}$ of beam current with a nominal beam diameter of $2 \mathrm{~nm}$. The exposure has been defined as a set of single exposures, with an e-beam dose of $225 \mu \mathrm{C} / \mathrm{cm}^{2}$ and line step size of $10 \mathrm{~nm}$.

After the exposure, the resist is developed and the sample is exposed to oxygen plasma to chemically modify the lithographically defined regions. The functionalization is performed in an Alcatel AMS 110 DE reactive ion etching (RIE) equipment by using two different conditions, referred in the text as mild (150 W, $10 \mathrm{sccm} \mathrm{O}$ for 5 seconds) and strong ( $300 \mathrm{~W}, 50 \mathrm{sccm} \mathrm{O}_{2}$ for 10 seconds). 
After performing the brush functionalization, the resist is removed. As revealed in the AFM images (topography and phase signals) of figure S1, when mild conditions are used, only some chemical contrast is observed (no topographical changes are seen), whereas when functionalization conditions are stronger, there are both topographical and chemical changes on the surface, thus promoting different orientations of the BCP.

Finally, the BCP is deposited from a $1.5 \%$ PGMEA solution at $2500 \mathrm{rpm}$, and annealed at $230^{\circ} \mathrm{C}$ for 10 min with a continuous nitrogen flow, in order to direct the self-assemble with respect to the previous chemical pattern.

\section{Simulations}

In our top-down coarse-grained model ${ }^{63}$ we describe the symmetric copolymers be a discretized EdwardsHamiltonian using $N=32$ beads per chain molecule. The molecules of the random copolymer brush are composed of $N_{r c}=8$ coarse-grained segments. This chain-length ratio between the copolymers and the brush molecules mimics the experimental system. Bonded beads interact via harmonic springs and the mean-squared end-to-end distance, $R_{e 0}$, of the copolymer (in the absence of non-bonded interactions) sets the unit of length of the computational model. Non-bonded interactions are catered for by the Hamiltonian:

$\frac{\mathcal{H}_{\mathrm{nb}}}{k_{\mathrm{B}} T \sqrt{\overline{\mathcal{N}}}}=\int_{V} \frac{\mathrm{d} \mathbf{r}}{R_{e 0}^{3}}\left\{\frac{\kappa N}{2}\left[\phi_{A}+\phi_{B}-1\right]^{2}-\frac{\chi N}{4}\left[\phi_{A}-\phi_{B}\right]^{2}-\frac{\Lambda N R_{e 0}}{\epsilon}\left(\hat{\phi}_{A}-\hat{\phi}_{B}\right) f(y, z) e^{-\frac{x^{2}}{2 \epsilon^{2}}}\right\}_{[2]}$

where $\overline{\mathcal{N}}$ denotes the invariant degree of polymerization, and $\phi_{A}$ and $\phi_{B}$ are the local, dimensionless densities of the A and B segments, respectively. The first term in Eq. [2] accounts for the small compressibility of a melt, and we use $\kappa N=50$ to set the inverse isothermal compressibility. The second term quantifies the repulsion between the different blocks of the copolymer, whose strength is set by the product $\chi N$ of the Flory-Huggins parameter and the number of segments per copolymer. The third term 
represents the short-range interaction between the components of the copolymer and the impenetrable substrate. For positive values of $\Lambda N$, the surface attracts the A component and repels the $\mathrm{B}$ segments of the copolymer. The interaction extends a distance $\varepsilon=0.15 R_{e}$ away from the surface into the film. $f(y, z)$ encodes the guiding pattern as a function of the two lateral coordinates, y and z. For the DSA simulation the top surface of the film is hard and non-preferential.

In single-chain-mean-field (SCMF) simulations the non-bonded interactions are temporarily replaced by fluctuating fields, which are frequently recomputed from the density distribution. The fields are computed by a linear assignment scheme on a collocation grid with spacing, $\Delta L \approx R_{e} / 6$. The molecular conformations are updated by a Smart-Monte-Carlo algorithm, which employs the strong bonded force to propose a trial displacement, resulting in a realistic Rouse-like dynamics. The "time" is takes a polymer to diffuse a distance $R_{e}$ is $\tau=8900$ SMC-steps. We use an invariant degree of polymerization $\overline{\mathcal{N}} \approx 90^{2}$ for the copolymers and $\chi N=17$ if not stated otherwise.

In order to calculate the macroscopic contact angle $\theta_{\mathrm{A}-\mathrm{B}}$ of a homopolymer blend, we simulate a symmetric homopolymer blend, $N_{A}=N_{B}=32$, in an anti-symmetric thin-film geometry, i.e., the top confining surface attracts the A segments with exactly the same strength as the bottom substrate attracts the B segments, i.e. $\Lambda N_{\text {top }}=-\Lambda N_{\text {bottom }}$ for the hard surface, and the $\mathrm{A}$ and $\mathrm{B}$ fractions of the random copolymer brush is switched,

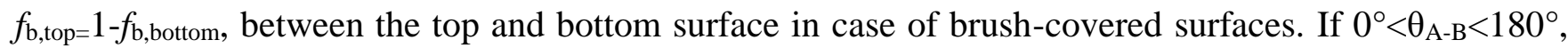
i.e., homopolymers do not wet the respective surface, the internal $\mathrm{AB}$ interface will run straight across the film (as shown in figure 4.a), and we can read off the macroscopic contact angle from the orientation of the $\mathrm{AB}$ interface in the middle of the film. ${ }^{64}$

In order to study DSA we start the simulation from a disordered configuration, $\chi N_{\text {initial }}=0$, instantaneously quench to $\chi \mathrm{N}=17$, and follow the kinetics of DSA. We use $L_{0}=1.67 R_{e 0}$. This value is slightly larger than the approximate estimate of the bulk lamellar spacing obtained from the isotropy of the virial of bonded forces in the bulk lamellae but it yields a broader parameter window for defect-free DSA. The snapshots 
depict the final, metastable structure at the end of the simulation that lasted longer than 400000 SMC steps $\approx 45 \tau$.

The simulated system for determination of the contact angle on hard surfaces is defined by: $\mathrm{N}=32, \chi \mathrm{N}$ $=16 ; 20$ and 30, and invariant degree of polymerization $\sqrt{\bar{N}}=n R_{e 0}^{3} / V=90$ and 128. The geometry of the simulated system is defined by $V=2.56 \times 10 \times 2 R_{e 0}^{3}$, discretization of collocation grid is $25 \times 6 \times 12$ with linear assignment between particle positions and grid points, hard walls on top and bottom, and antisymmetric surface fields of strength $\Lambda N$.

In order to calculate the macroscopic contact angle $\theta_{\mathrm{AB}}$ of a homopolymer blend, we simulate a symmetric homopolymer blend, $N_{A}=N_{B}=32$, in an anti-symmetric thin-film geometry, where the A and B fractions

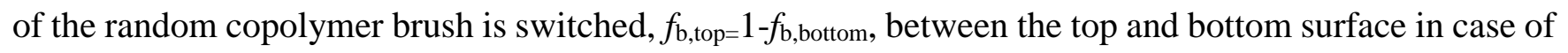
brush-covered surfaces. If $0^{\circ}<\theta_{\mathrm{AB}}<180^{\circ}$, i.e., homopolymers do not wet the respective surface, the internal $\mathrm{AB}$ interface will run straight across the film (as shown in Figure 4.a), and we can read off the macroscopic contact angle from the orientation of the AB interface in the middle of the film. ${ }^{64}$

\section{CONCLUSIONS}

In summary, we have shown that the interpenetration between the copolymer brush on the brush substrate influences the final morphology of the block copolymer patterns in directed self-assembly by chemical epitaxy when using wide guiding stripes. Experimental results have been quantitatively compared to computer simulations using the experimentally determined contact angle of the interface of the polymer components with the substrate surface to characterize the surface affinity. Simulations and experiments only agree if the responsive, penetrable structure of the copolymer brush on the substrate is accounted for. This observation indicates that the surface free energy difference between the components is not the only quantity that the top-down coarse-grained model requires in order to quantitatively describe the experimental system. In consequence, not only the composition of the brush layer dictates the surface 
affinity, but also structural parameters like its grafting density must be considered to achieve an optimal DSA performance.

\section{SUPPORTING INFORMATION}

AFM images of chemical guiding patterns; Values of the difference of surface free-energies for various brush-block copolymer systems; SEM images of directed self-assembly of block copolymers of different pitch by using chemical wide patterns; Water contact angle values on different modified and un-modified brush layers; SEM images of PS-b-PMMA $($ L0 = $22 \mathrm{~nm})$ on top of various brush layers; Values of experimental surface free energies obtained by using the sessile drop method for different brush layer materials; Simulations of the same system as in Figure 4.c but with an increased strength of the shortrange potential of the guiding pattern;

\section{ACKNOWLEDGMENTS}

This work was partially funded by the projects CoLiSA.MMP (ICT Project no. 619793) and NANOINTEGRA (TEC2015-69864-R, MINECO/FEDER, UE). Laura Evangelio acknowledges the Ministry of Education of Spain for her FPU grant (FPU 13/03746). Marcus Müller thanks the Deutsche Forschungsgemeinschaft for financial support under grant Mu1674/16. The ICN2 is funded by the CERCA programme / Generalitat de Catalunya. The ICN2 is supported by the Severo Ochoa programme of the Spanish Ministry of Economy, Industry and Competitiveness (MINECO, grant no. SEV-20130295).

\section{REFERENCES}

(1) Moore, G. E. Craming More Components onto Integrated Circuits. Electronics 1965, 38 (8), 114 117. 
(2) Mansky, P.; haikin, P.; Thomas, E. L. Monolayer Films of Diblock Copolymer Microdomains for Nanolithographic Applications. J. Mater. Sci. 1995, 30 (8), 1987-1992.

(3) Ruiz, R.; Kang, H.; Detcheverry, F. A.; Dobisz, E.; Kercher, D. S.; Albrecht, T. R.; De Pablo, J. J.; Nealey, P. F. Density Multiplication and Improved Lithography by Directed Block Copolymer Assembly. Science (80-. ). 2008, 321 (5891), 936-939.

(4) Liu, G.; Thomas, C. S.; Craig, G. S. W.; Nealey, P. F. Integration of Density Multiplication in the Formation of Device-Oriented Structures by Directed Assembly of Block CopolymerHomopolymer Blends. Adv. Funct. Mater. 2010, 20 (8), 1251-1257.

(5) Rathsack, B.; Somervell, M.; Muramatsu, M.; Tanouchi, K.; Kitano, T.; Nishimura, E.; Yatsuda, K.; Nagahara, S.; Iwaki, H.; Akai, K.; Ozawa, M.; Romo Negreira, A.; Tahara, S.; Nafus, K. Advances in Directed Self Assembly Integration and Manufacturability at $300 \mathrm{Mm}$. Proc. SPIE 2013, 8682 (March 2013), 86820K.

(6) Cheng, J. Y.; Rettner, C. T.; Sanders, D. P.; Kim, H.-C.; Hinsberg, W. D. Dense Self-Assembly on Sparse Chemical Patterns: Rectifying and Multiplying Lithographic Patterns Using Block Copolymers. Adv. Mater. 2008, 20 (16), 3155-3158.

(7) Stoykovich, M. P.; Daoulas, K. C.; Müller, M.; Kang, H.; de Pablo, J. J.; Nealey, P. F. Remediation of Line Edge Roughness in Chemical Nanopatterns by the Directed Assembly of Overlying Block Copolymer Films. Macromolecules 2010, 43 (5), 2334-2342.

(8) Wan, L.; Ruiz, R.; Gao, H.; Patel, K. C.; Albrecht, T. R.; Yin, J.; Kim, J.; Cao, Y.; Lin, G. The Limits of Lamellae-Forming PS- $b$-PMMA Block Copolymers for Lithography. ACS Nano 2015, $9(7), 7506-7514$.

(9) Mansky, P.; Liu, Y.; Huang, E.; Russell, T. P.; Hawker, C. Controlling Polymer-Surface Interactions with Random Copolymer Brushes. Science (80-. ). 1997, 275 (5305), 1458-1460. 
(10) Roth, S. V.; Santoro, G.; Risch, J. F. H.; Yu, S.; Schwartzkopf, M.; Boese, T.; Döhrmann, R.; Zhang, P.; Besner, B.; Bremer, P.; Rukser, D.; Rübhausen, M. A.; Terrill, N. J.; Staniec, P. A.; Yao, Y.; Metwalli, E.; Müller-Buschbaum, P. Patterned Diblock Co-Polymer Thin Films as Templates for Advanced Anisotropic Metal Nanostructures. ACS Appl. Mater. Interfaces 2015, 7 (23), 1247012477.

(11) Han, E.; Kang, H.; Liu, C.-C.; Nealey, P. F.; Gopalan, P. Graphoepitaxial Assembly of Symmetric Block Copolymers on Weakly Preferential Substrates. Adv. Mater. 2010, 22 (38), 4325-4329.

(12) Servin, I.; Tiron, R.; Gharbi, A.; Argoud, M.; Jullian, K.; Chamiot-Maitral, G.; Barros, P. P.; Chevalier, X.; Belledent, J.; Bossy, X.; Moulis, S.; Navarro, C.; Cunge, G.; Barnola, S.; Asai, M.; Pieczulewski, C. Contact Hole Shrink by Directed Self-Assembly: Process Integration and Stability Monitored on 300mm Pilot Line. Jpn. J. Appl. Phys. 2014, 53 (6 SPEC. ISSUE), 1-6.

(13) Han, E.; Kang, H.; Liu, C. C.; Nealey, P. F.; Gopalan, P. Graphoepitaxial Assembly of Symmetric Block Copolymers on Weakly Preferential Substrates. Adv. Mater. 2010, 22 (38), 4325-4329.

(14) Cheng, J. Y.; Ross, C. a.; Thomas, E. L.; Smith, H. I.; Vancso, G. J. Fabrication of Nanostructures with Long-Range Order Using Block Copolymer Lithography. Appl. Phys. Lett. 2002, 81 (19), 3657.

(15) Park, S.; Lee, D. H.; Xu, J.; Kim, B.; Hong, S. W.; Jeong, U.; Xu, T.; Russell, T. P. Macroscopic 10-Terabit-per-Square-Inch Arrays from Block Copolymers with Lateral Order. Science 2009, 323 (February), 1030-1033.

(16) Tiron, R.; Chevalier, X.; Gaugiran, S.; Pradelles, J.; Fontaine, H.; Couderc, C.; Pain, L.; Navarro, C.; Chevolleau, T.; Cunge, G.; Delalande, M.; Fleury, G.; Hadziioannou, G. Pattern Density Multiplication by Direct Self Assembly of Block Copolymers: Toward 300mm CMOS Requirements. 2012, 8323, 832300 . 
(17) Kim, S. O.; Solak, H. H.; Stoykovich, M. P.; Ferrier, N. J.; De Pablo, J. J.; Nealey, P. F. Epitaxial Self-Assembly of Block Copolymers on Lithographically Defined Nanopatterned Substrates. Nature 2003, 424 (6947), 411-414.

(18) Edwards, E. W.; Stoykovich, M. P.; Solak, H. H.; Nealey, P. F. Long-Range Order and Orientation of Cylinder-Forming Block Copolymers on Chemically Nanopatterned Striped Surfaces. Macromolecules 2006, 39 (10), 3598-3607.

(19) Liu, C. C.; Ramírez-Hernández, A.; Han, E.; Craig, G. S. W.; Tada, Y.; Yoshida, H.; Kang, H.; Ji, S.; Gopalan, P.; De Pablo, J. J.; Nealey, P. F. Chemical Patterns for Directed Self-Assembly of Lamellae-Forming Block Copolymers with Density Multiplication of Features. Macromolecules 2013, 46 (4), 1415-1424.

(20) Stoykovich, M. P.; Nealey, P. F. Block Copolymers and Conventional Lithography. Mater. Today 2006, 9 (9), 20-29.

(21) Liu, C.-C.; Han, E.; Onses, M. S.; Thode, C. J.; Ji, S.; Gopalan, P.; Nealey, P. F. Fabrication of Lithographically Defined Chemically Patterned Polymer Brushes and Mats. Macromolecules 2011, $44(7), 1876-1885$.

(22) Kim, J.; Wan, J.; Miyazaki, S.; Yin, J.; Cao, Y.; Her, Y. J.; Wu, H.; Shan, J.; Kurosawa, K.; Lin, G. The SMARTTM Process for Directed Block Co-Polymer Self-Assembly. J. Photopolym. Sci. Technol. 2013, 26 (5), 573-579.

(23) Ji, S.; Nagpal, U.; Liu, G.; Delcambre, S. P.; Müller, M.; De Pablo, J. J.; Nealey, P. F. Directed Assembly of Non-Equilibrium ABA Triblock Copolymer Morphologies on Nanopatterned Substrates. ACS Nano 2012, 6 (6), 5440-5448.

(24) Cushen, J.; Wan, L.; Blachut, G.; Maher, M. J.; Albrecht, T. R.; Ellison, C. J.; Willson, C. G.; Ruiz, R. Double-Patterned Sidewall Directed Self-Assembly and Pattern Transfer of Sub-10 Nm 
PTMSS- b-PMOST. ACS Appl. Mater. Interfaces 2015, 7 (24), 13476-13483.

(25) Williamson, L. D.; Seidel, R. N.; Chen, X.; Suh, H. S.; Rincon Delgadillo, P.; Gronheid, R.; Nealey, P. F. Three-Tone Chemical Patterns for Block Copolymer Directed Self-Assembly. ACS Appl. Mater. Interfaces 2016, 8 (4), 2704-2712.

(26) Khaira, G. S.; Qin, J.; Garner, G. P.; Xiong, S.; Wan, L.; Ruiz, R.; Jaeger, H. M.; Nealey, P. F.; De Pablo, J. J. Evolutionary Optimization of Directed Self-Assembly of Triblock Copolymers on Chemically Patterned Substrates. ACS Macro Lett. 2014, 3 (8), 747-752.

(27) Xiong, S.; Wan, L.; Ishida, Y.; Chapuis, Y. A.; Craig, G. S. W.; Ruiz, R.; Nealey, P. F. Directed Self-Assembly of Triblock Copolymer on Chemical Patterns for Sub-10-Nm Nanofabrication via Solvent Annealing. ACS Nano 2016, 10 (8), 7855-7865.

(28) Blachut, G.; Sirard, S. M.; Maher, M. J.; Asano, Y.; Someya, Y.; Lane, A. P.; Durand, W. J.; Bates, C. M.; Dinhobl, A. M.; Gronheid, R.; Hymes, D.; Ellison, C. J.; Willson, C. G. A Hybrid Chemo/Grapho-Epitaxial Alignment Strategy for Defect Reduction in Sub-10 Nm Directed Self-Assembly of Silicon-Containing Block Copolymers. Chem. Mater. 2016, 28 (24), 8951-8961.

(29) Segal-Peretz, T.; Ren, J.; Xiong, S.; Khaira, G.; Bowen, A.; Ocola, L. E.; Divan, R.; Doxastakis, M.; Ferrier, N. J.; de Pablo, J.; Nealey, P. F. Quantitative Three-Dimensional Characterization of Block Copolymer Directed Self-Assembly on Combined Chemical and Topographical Prepatterned Templates. ACS Nano 2017, 11 (2), 1307-1319.

(30) Evangelio, L.; Gramazio, F.; Lorenzoni, M.; Gorgoi, M.; Espinosa, F. M.; García, R.; PérezMurano, F.; Fraxedas, J. Identifying the Nature of Surface Chemical Modification for Directed Self-Assembly of Block Copolymers. Beilstein J. Nanotechnol. 2017, 8 (1), 1972-1981.

(31) Müller, M.; Smith, G. D. Phase Separation in Binary Mixtures Containing Polymers: A Quantitative Comparison of Single-Chain-in-Mean-Field Simulations and Computer Simulations 
of the Corresponding Multichain Systems. J. Polym. Sci. Part B Polym. Phys. 2005, 43 (8), 934958.

(32) Daoulas, K. C.; Müller, M.; de Pablo, J. J.; Nealey, P. F.; Smith, G. D. Morphology of MultiComponent Polymer Systems: Single Chain in Mean Field Simulation Studies. Soft Matter 2006, $2(7), 573-583$.

(33) Wang, J.; Müller, M. Microphase Separation of Diblock Copolymer Brushes in Selective Solvents: Single-Chain-in-Mean-Field Simulations and Integral Geometry Analysis. Macromolecules 2009, $42(6), 2251-2264$.

(34) Steinmüller, B.; Müller, M.; Hambrecht, K. R.; Smith, G. D.; Bedrov, D. Properties of Random Block Copolymer Morphologies: Molecular Dynamics and Single-Chain-in-Mean-Field Simulations. Macromolecules 2012, 45 (2), 1107-1117.

(35) Sparnacci, K.; Antonioli, D.; Perego, M.; Giammaria, T. J.; Seguini, G.; Ferrarese Lupi, F.; Zuccheri, G.; Gianotti, V.; Laus, M. High Temperature Surface Neutralization Process with Random Copolymers for Block Copolymer Self-Assembly. Polym. Int. 2017, 66 (3), 459-467.

(36) Sparnacci, K.; Antonioli, D.; Gianotti, V.; Laus, M.; Ferrarese Lupi, F.; Giammaria, T. J.; Seguini, G.; Perego, M. Ultrathin Random Copolymer-Grafted Layers for Block Copolymer Self-Assembly. ACS Appl. Mater. Interfaces 2015, 7 (20), 10944-10951.

(37) Wolkenhauer, M.; Bumbu, G. G.; Cheng, Y.; Roth, S. V.; Gutmann, J. S. Investigation of Micromechanical Cantilever Sensors with Microfocus Grazing Incidence Small-Angle x-Ray Scattering. Appl. Phys. Lett. 2006, 89 (5), 054101.

(38) Durand, W. J.; Carlson, M. C.; Maher, M. J.; Blachut, G.; Santos, L. J.; Tein, S.; Ganesan, V.; Ellison, C. J.; Willson, C. G. Experimental and Modeling Study of Domain Orientation in Confined Block Copolymer Thin Films. Macromolecules 2016, 49 (1), 308-316. 
(39) Turner, M. S. Equilibrium Properties of a Diblock Copolymer Lamellar Phase Confined between Flat Plates. Phys. Rev. Lett. 1992, 69 (12), 1788-1791.

(40) Geisinger, T.; Müller, M.; Binder, K. Symmetric Diblock Copolymers in Thin Films (I): Phase Stability in Self-Consistent Field Calculations and Monte Carlo Simulations. J. Chem. Phys. 1999, $111(11), 5241-5250$.

(41) Johnson, W. C.; Wang, J.; Chen, Z. Surface Structures and Properties of Polystyrene / Poly ( Methyl Methacrylate ) Blends and Copolymers. J. Phys. Chem. B 2005, 109, 6280-6286.

(42) Ahn, D. U.; Wang, Z.; Campbell, I. P.; Stoykovich, M. P.; Ding, Y. Morphological Evolution of Thin PS/PMMA Films: Effects of Surface Energy and Blend Composition. Polym. (United Kingdom) 2012, 53 (19), 4187-4194.

(43) Vitt, E.; Shull, K. R. Equilibrium Contact Angle for Polymer/Polymer Interfaces. Macromolecules 1995, 28 (18), 6349-6353.

(44) Carriere, C. J.; Biresaw, G.; Sammler, R. L. Temperature Dependence of the Interfacial Tension of PS / PMMA , PS / PE , and PMMA / PE Blends. Rheol. Acta 2000, 39, 476-482.

(45) Peters, R. D.; Yang, X. M.; Kim, T. K.; Sohn, B. H.; Nealey, P. F. Using Self-Assembled Monolayers Exposed to X-Rays to Control the Wetting Behavior of Thin Films of Diblock Copolymers. Langmuir 2000, 16 (10), 4625-4631.

(46) Mansky, P.; Russell, T. P.; Hawker, C. J.; Pitsikalis, M.; Mays, J. Ordered Diblock Copolymer Films on Random Copolymer Brushes. Macromolecules 1997, 30 (22), 6810-6813.

(47) Kim, S.; Bates, C. M.; Thio, A.; Cushen, J. D.; Ellison, C. J.; Willson, C. G.; Bates, F. S. Consequences of Surface Neutralization in Diblock Copolymer Thin Films. ACS Nano 2013, 7 (11), 9905-9919. 
(48) Maher, M. J.; Self, J. L.; Stasiak, P.; Blachut, G.; Ellison, C. J.; Matsen, M. W.; Bates, C. M.; Willson, C. G. Structure, Stability, and Reorganization of 0.5 L0 Topography in Block Copolymer Thin Films. ACS Nano 2016, 10 (11), 10152-10160.

(49) Owens, D. K.; Wendt, Y. Estimation of the Surface Free Energy of Polymers. J. Appl. Polym. Sci. 1969, 13, 1741-1747.

(50) Edwards, E. W.; Mu, M.; Stoykovich, M. P.; Solak, H. H.; Pablo, J. J. De; Nealey, P. F. Dimensions and Shapes of Block Copolymer Domains Assembled on Lithographically Defined Chemically Patterned Substrates. 2007, 90-96.

(51) Semenov, A.; Theory, A. S.; Interactions, L.; Journal, S.; Ii, D. P. Theory of Long-Range Interactions in Polymer Systems To Cite This Version : Long-Range ( I ) J. 1996, 6 (12), 17591780.

(52) Pandav, G.; Durand, W. J.; Ellison, C. J.; Willson, C. G.; Ganesan, V. Directed Self Assembly of Block Copolymers Using Chemical Patterns with Sidewall Guiding Lines, Backfilled with Random Copolymer Brushes. Soft Matter 2015, 11 (47), 9107-9114.

(53) Trombly, D. M.; Pryamitsyn, V.; Ganesan, V. Surface Energies and Self-Assembly of Block Copolymers on Grafted Surfaces. Phys. Rev. Lett. 2011, 107 (14), 148304.

(54) Song, J.-Q.; Liu, Y. X.; Zhang, H.-D. J. A Surface Interaction Model for Self-Assembly of Block Copolymers under Soft Confinement. J. Chem. Phys. 2016, 145 (21), 214902.

(55) Leibler, L.; Ajdari, A.; Mourran, A.; Coulon, G.; Chatenay, D. Wetting of Grafted Polymer Surfaces by Compatible Chains. In Ordering in Macromolecular Systems: Proceedings of the OUMS'93 Toyonaka, Osaka, Japan, 3--6 June 1993; Teramoto, A., Kobayashi, M., Norisuye, T., Eds.; Springer Berlin Heidelberg: Berlin, Heidelberg, 1994; pp 301-311. 
(56) Shull, K. R. Wetting Autophobicity of Polymer Melts. Faraday Discuss. 1994, 98 (0), $203-217$.

(57) Stoykovich, M. P.; Kang, H.; Daoulas, K. C.; Liu, G.; Liu, C. C.; De Pablo, J. J.; Müller, M.; Nealey, P. F. Directed Self-Assembly of Block Copolymers for Nanolithography: Fabrication of Isolated Features and Essential Integrated Circuit Geometries. ACS Nano 2007, 1 (3), 168-175.

(58) Shin, D. O.; Kim, B. H.; Kang, J.-H.; Jeong, S.-J.; Park, S. H.; Lee, Y.-H.; Kim, S. O. OneDimensional Nanoassembly of Block Copolymers Tailored by Chemically Patterned Surfaces. Macromolecules 2009, 42 (4), 1189-1193.

(59) Kim, B. H.; Byeon, K. J.; Kim, J. Y.; Kim, J.; Jin, H. M.; Cho, J. Y.; Jeong, S. J.; Shin, J.; Lee, H.; Kim, S. O. Negative-Tone Block Copolymer Lithography by in Situ Surface Chemical Modification. Small 2014, 10 (20), 4207-4212.

(60) Tamulevicius, S.; Prosycevas, I.; Guobienè, A.; Puiso, J. Oxygen Plasma Processing of Silicon and Silica Substrates for Thin Films of Polymer Blends. Solid State Phenom. 2004, 99-100, 175-180.

(61) Kaya, S.; Rajan, P.; Dasari, H.; Ingram, D. C.; Jadwisienczak, W.; Rahman, F. A Systematic Study of Plasma Activation of Silicon Surfaces for Self Assembly. ACS Appl. Mater. Interfaces 2015, 7 (45), 25024-25031.

(62) Onses, M. S.; Ramírez-Hernández, A.; Hur, S. M.; Sutanto, E.; Williamson, L.; Alleyne, A. G.; Nealey, P. F.; De Pablo, J. J.; Rogers, J. A. Block Copolymer Assembly on Nanoscale Patterns of Polymer Brushes Formed by Electrohydrodynamic Jet Printing. ACS Nano 2014, 8 (7), 6606-6613.

(63) Müller, M. Studying Amphiphilic Self-Assembly with Soft Coarse-Grained Models. J. Stat. Phys. 2011, 145 (4), 967-1016.

(64) Müller, M.; Albano, E. V; Binder, K. Symmetric Polymer Blend Confined into a Film with Antisymmetric Surfaces: Interplay between Wetting Behavior and the Phase Diagram. Phys. Rev. 
E 2000, 62 (4), 5281-5295. 


\section{FIGURES}

(a)
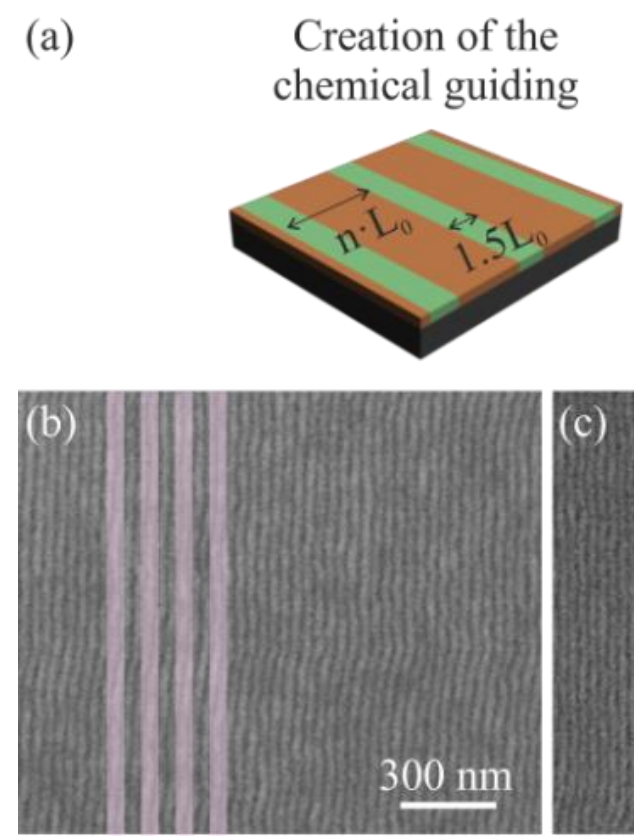

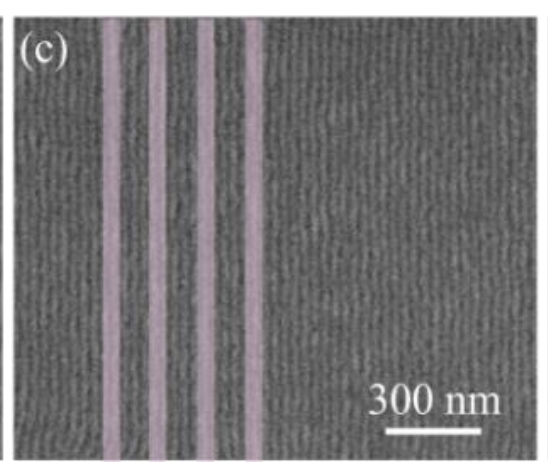

PS- $b$-PMMA

directed self-assembly
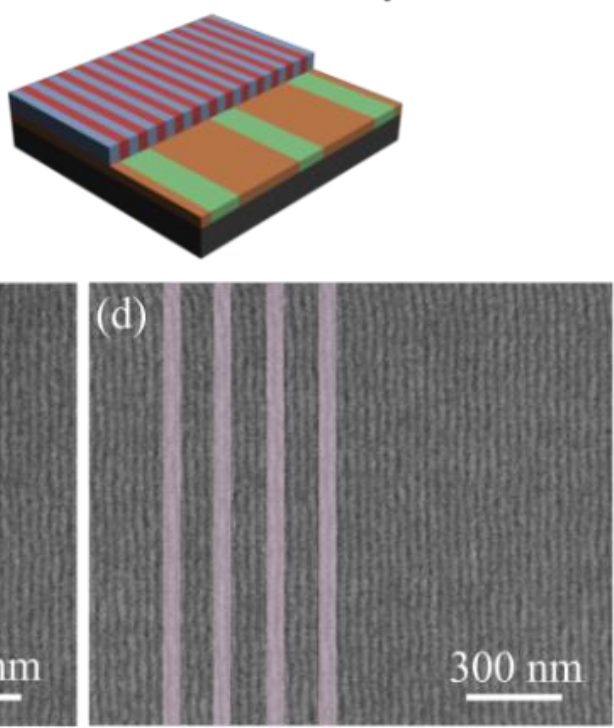

Figure 1. (a) Schematic representation of DSA on chemical wide patterns using EBL and oxygen plasma functionalization. SEM images of PS-b-PMMA DSA on $1.5 \mathrm{~L}_{0}$ width guiding stripes and density multiplication factors of (b) $3 \mathrm{~L}_{0}$, (c) $4 \mathrm{~L}_{0}$ and (d) $5 \mathrm{~L}_{0}$ (red stripes represent the guiding stripe width). 

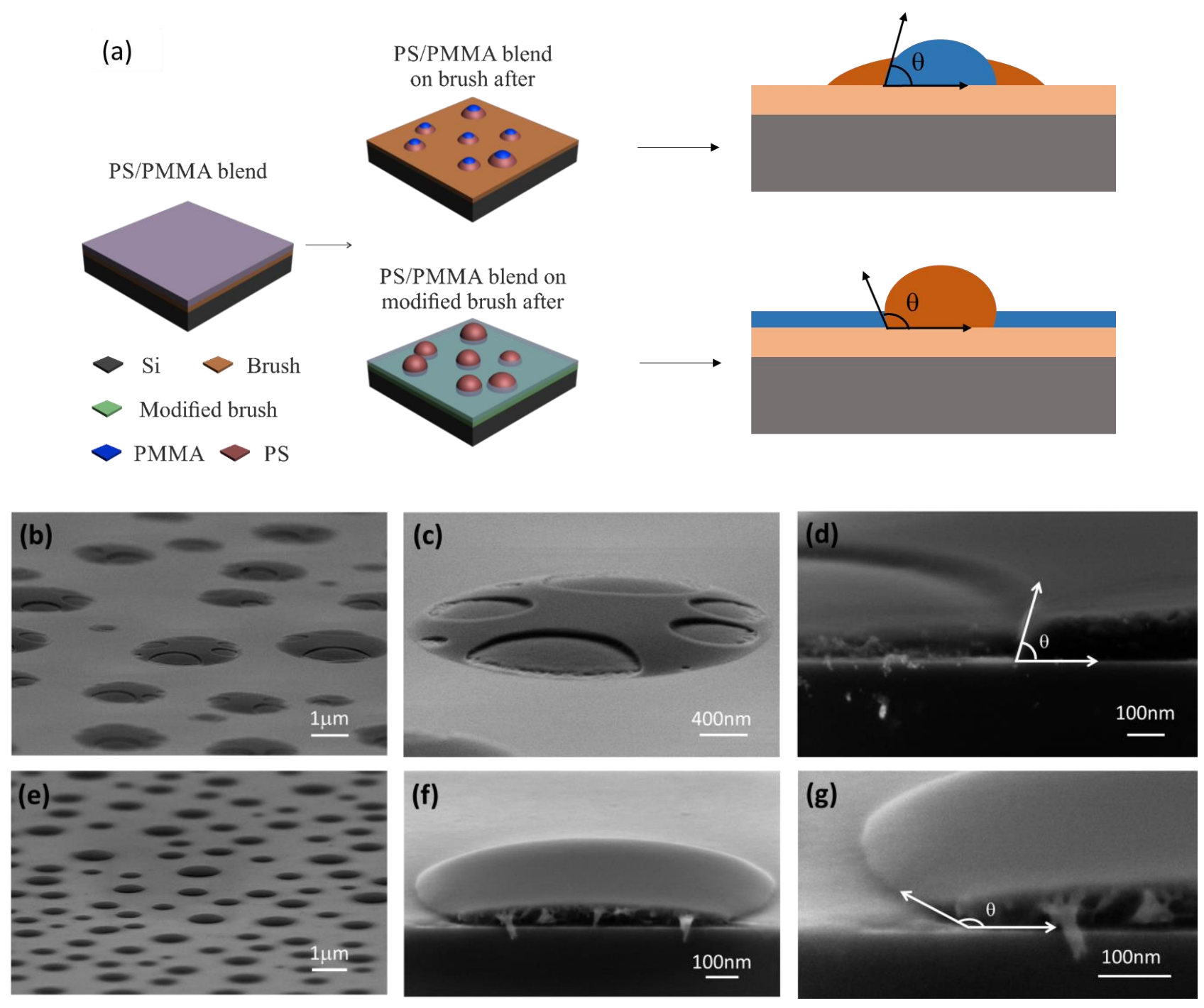

Figure 2. (a): Scheme of the method to determine the contact angle between homopolymers in unmodified (top) and modified (bottom) brush surfaces. Droplets are obtained by dewetting of homopolymer blends under the same annealing conditions than the ones used for DSA. (b-g): SEM images showing the droplets of polymer blends after dewetting and after being removed the PMMA domains by $\mathrm{O}_{2}$ plasma ( $260 \mathrm{~W}, 50 \mathrm{sccm} \mathrm{O}_{2}$ for 18 seconds); SEM images in (b-d) corresponds to a dewetting experiment on an un-modified brush; SEM images in (e-g) corresponds to the modified brush. In both cased the brush is PS70\%-r-PMMA 
(a)
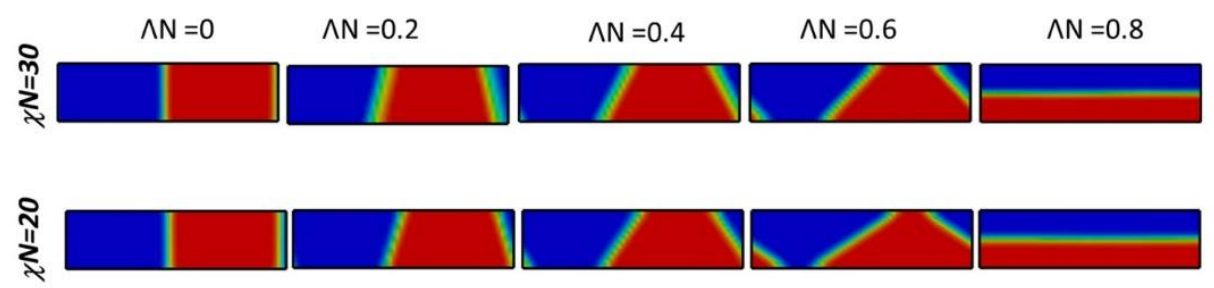

(b)

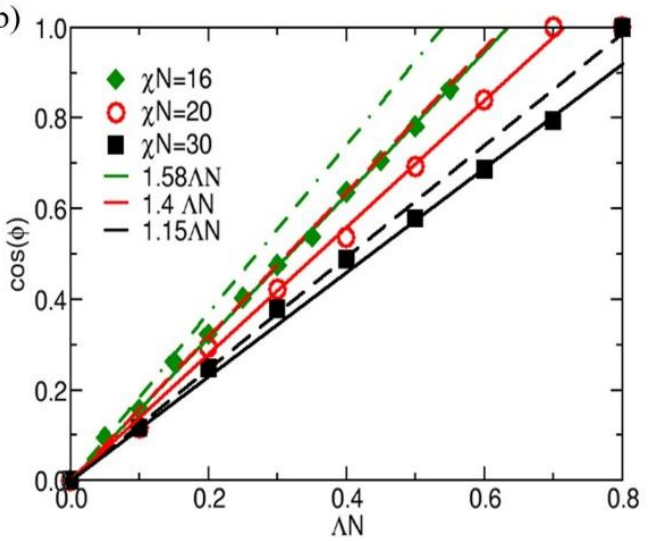

(c)

hard surface/short-range potential $\Lambda N=0.538$ (guide) $/-0.135(\mathrm{bg})$

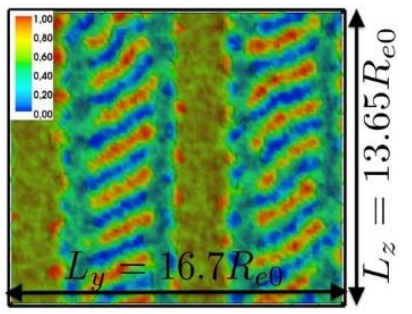

Figure 3. (a) Examples of simulation of symmetric homopolymer blends for two values of $\chi N$ as a function of the surface strength, $\Lambda N$. (b) Relation between contact angle $\theta_{A B}$ and surface strength $\Lambda \mathrm{N}$ from the analytical prediction (dashed lines) and from simulations (c) Front and top-down views of SCMF simulation for a thin symmetric BCP film. Results for a hard film surface with a short-range attraction, $\Lambda N=0.538$ for the narrow guiding stripe with width $1.5 L_{0}$ and $\Lambda N=-0.135$ for the background with width $3.5 L_{0}$. The film thickness is $D=1.13 R_{e} 0$. 

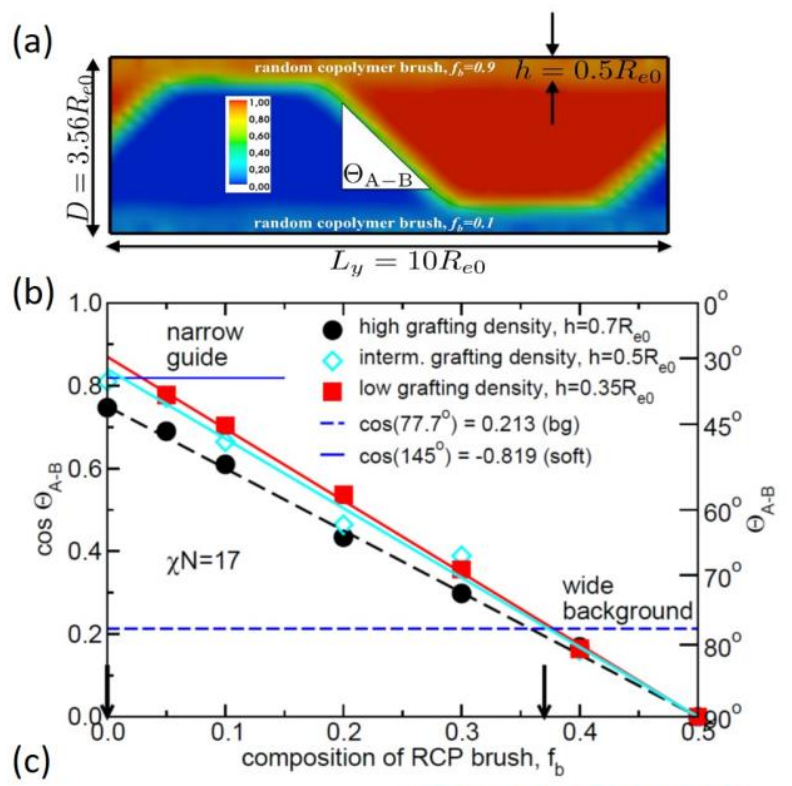

(c)

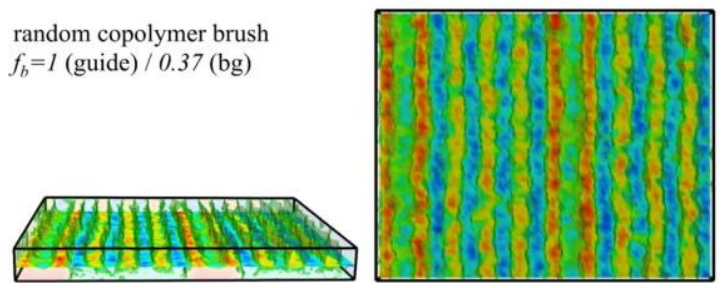

Figure 4. a) Two-dimensional composition profile of a homopolymer blend, $N=32$, confined between two antisymmetric random-copolymer brushes with A fractions, $f_{b}=0.1$ and 0.9 . The system geometry is $3.56 R_{e 0} \times 10 R_{e 0} \times 2 R_{e 0}$ and the system is comprised of 2304 A homopolymers, 2304 B homopolymers and 7200 grafted random copolymers that are a factor 4 shorter. The white wedge indicates the contact angle that the internal $\mathrm{AB}$ interface makes with the confining surfaces in the middle of the film. (b) Contact angle, $\theta_{A-B}$, of a symmetric homopolymer blend with $N=32$ on a random-copolymer brush with $N_{r c}=8$ as a function of the composition, $f_{b} \cdot \chi N=17$ and $\overline{\mathcal{N}} \approx 90^{2}$. The horizontal lines mark the contact angle on the wide background stripe and the symmetry-related value of the narrow guiding stripe corresponding to the experimental sample $\mathrm{PS}_{70 \%-\mathrm{r}-}$ PMMA. The arrows on the abscissa indicate the compositions of the random copolymer brush, $f_{b}=0.37$ (background) and $1-f_{b}=0$ (guiding stripe). (c) Front and top-down views of SCMF simulation for a thin symmetric BCP film for a brush-coated surface with effective brush thickness $h=0.5 R_{e}$ and total film thickness $D=1.64 R_{e} 0$. The composition of the random copolymer brush $f_{b}$ is 1 (i.e., A-homopolymer brush) and 0.37 on the narrow guiding stripes and the wide background, respectively. 

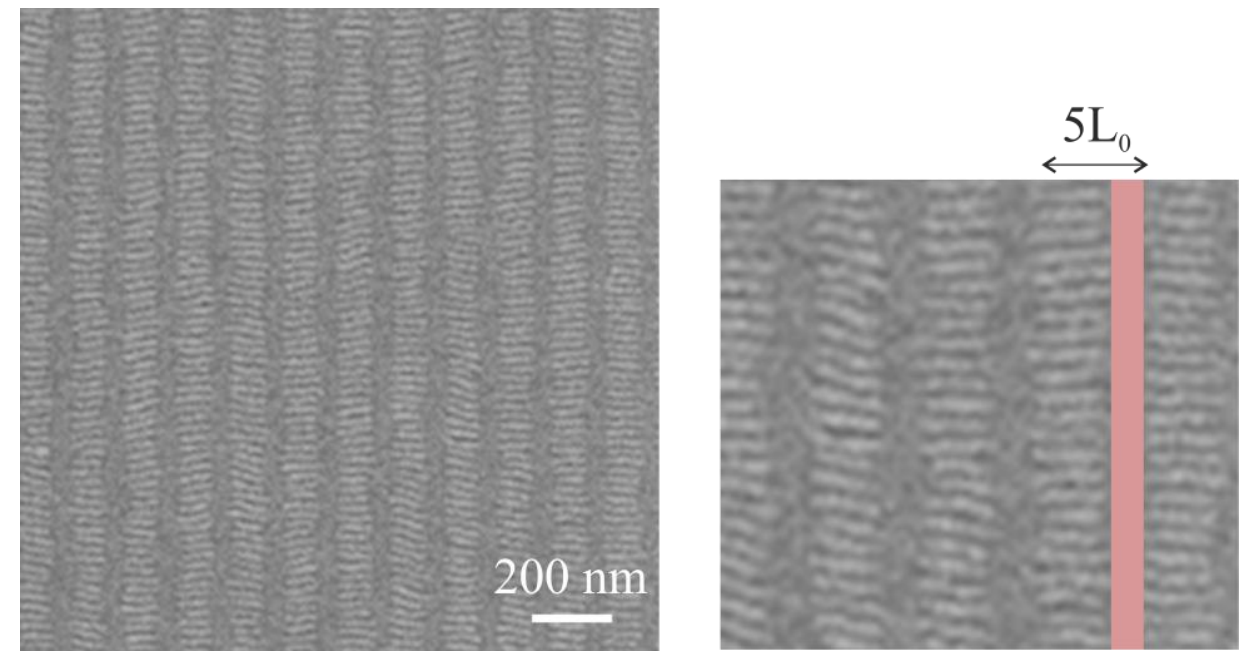

Figure 5. SEM images of the result of directed self-assembly when the chemical guiding patterns are formed using strong oxygen plasma conditions $\left(300 \mathrm{~W}, 50 \mathrm{sccm} \mathrm{O}_{2}\right.$ for $\left.10 \mathrm{~s}\right)$. The block copolymer is PS-b-PMMA that provides a pitch $\mathrm{L}_{0}=22 \mathrm{~nm}$. The width of the chemical guiding stripes dimensions is $1.5 \mathrm{~L}_{0}$ and the period is $5 \mathrm{~L}_{0}$. The right image is a zoom-in on the right image to better appreciate the parallel alignment of the block copolymer on the chemically modified stripes. 


\begin{tabular}{|c|c|c|c|c|c|c|}
\hline \multirow{2}{*}{ System } & \multicolumn{3}{|c|}{ PS6\%-r-PMMA } & \multicolumn{3}{c|}{ PS70\%-PMMA } \\
\cline { 2 - 7 } & $\begin{array}{c}\text { Un- } \\
\text { modified }\end{array}$ & $\begin{array}{c}\text { Mild } \mathrm{O}_{2} \\
\text { plasma }\end{array}$ & $\begin{array}{c}\text { Strong } \\
\mathrm{O}_{2} \\
\text { plasma }\end{array}$ & $\begin{array}{c}\text { Un- } \\
\text { modified }\end{array}$ & $\begin{array}{c}\text { Mild } \mathrm{O}_{2} \\
\text { plasma }\end{array}$ & $\begin{array}{c}\text { Strong } \\
\mathrm{O}_{2} \\
\text { plasma }\end{array}$ \\
\hline$\theta$ & $107.7^{\circ}$ & $154.5^{\circ}$ & $159.7^{\circ}$ & $77.7^{\circ}$ & $145^{\circ}$ & $155.8^{\circ}$ \\
\hline $\cos \theta$ & -0.30 & -0.90 & -0.94 & 0.21 & -0.82 & -0.91 \\
\hline$\Delta \gamma(\mathrm{mN} / \mathrm{m})$ & -0.18 & -0.55 & -0.57 & 0.13 & -0.50 & -0.56 \\
\hline \multirow{2}{*}{ System } & PS8\%-r-PMMA & \multicolumn{3}{c|}{ PS-OH } \\
\cline { 2 - 8 } & $\begin{array}{c}\text { Un- } \\
\text { modified }\end{array}$ & $\begin{array}{c}\text { Mild } \mathrm{O}_{2} \\
\text { plasma }\end{array}$ & $\begin{array}{c}\text { Strong } \\
\mathrm{O}_{2}\end{array}$ & $\begin{array}{c}\text { Un- } \\
\text { plasma }\end{array}$ & $\begin{array}{c}\text { Mild } \mathrm{O}_{2} \\
\text { plasma }\end{array}$ & $\begin{array}{c}\text { Strong } \\
\mathrm{O}_{2} \\
\text { plasma }\end{array}$ \\
\hline$\theta$ & $64.9^{\circ}$ & $141.6^{\circ}$ & $155.3^{\circ}$ & $55^{\circ}$ & $128^{\circ}$ & $147.1^{\circ}$ \\
\hline $\cos \theta$ & 0.42 & -0.78 & -0.91 & 0.57 & -0.62 & -0.84 \\
\hline$\Delta \gamma(\mathrm{mN} / \mathrm{m})$ & 0.26 & -0.48 & -0.56 & 0.35 & -0.38 & -0.51 \\
\hline
\end{tabular}

Table 1. Values of experimental difference of surface free energies obtained from homopolymer blend contact angle measurements for different brush layer materials. The values are calculated for the conditions to process PS-b-PMMA $\left(\mathrm{T}=230^{\circ} \mathrm{C}\right.$, i.e., $\left.\gamma_{\mathrm{PS} / \mathrm{PMMA}}=0.61 \mathrm{mN} / \mathrm{m}\right)$. For each brush layer (i.e., different fraction of PS), the contact angles are determined for the as grafted surface, and for two different conditions of oxygen plasma exposure to chemically modidy its character. 
TABLE OF CONTENTS GRAPHIC

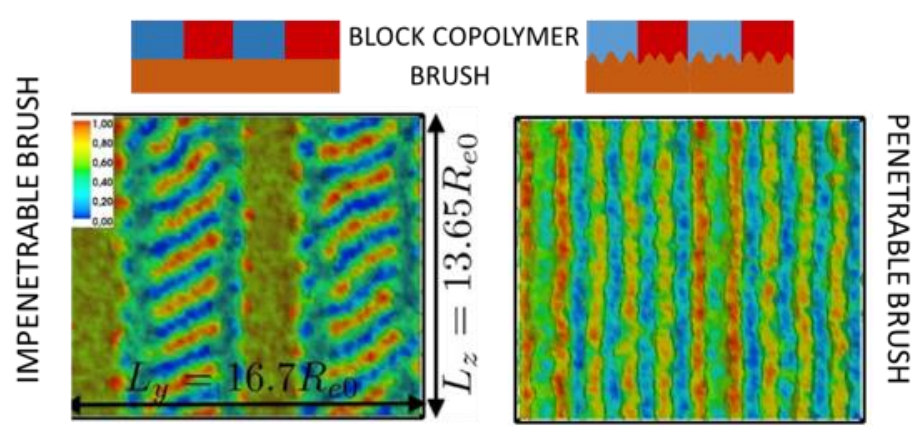

